\title{
Issues Under Negotiation
}

\author{
Staffan Larsson \\ Dept. of linguistics, Box 200 \\ Göteborg University \\ SE 40530 Göteborg, Sweden \\ sl@ling.gu.se
}

\begin{abstract}
In this paper, we give an account of a simple kind of collaborative negotiative dialogue. We also sketch a formalization of this account and discuss its implementation in a dialogue system.
\end{abstract}

\section{Introduction}

In this paper, we give an issue-based account of a simple kind of collaborative negotiative dialogue ${ }^{1}$. We start from a previous formal account of negotiative dialogue (Sidner, 1994a) and argue for a slightly different idea of what negotiative dialogue is. We want to make a distinction between the process of accepting an utterance and its content, which applies to all utterances, and a concept of negotiation defined, roughly, as a discussion of several alternative solutions to some problem. This latter account is formulated in terms of Issues Under Negotiation (IUN), representing the question or problem to be resolved, and a set of alternative answers, representing the proposed solutions. We argue that this theory improves on certain aspects of Sidner's account while also fitting into a question-based dialogue management framework which has previously been implemented in the GoDiS dialogue system (Bohlin et al., 1999).

\footnotetext{
${ }^{1}$ Work on this paper was supported by SIRIDUS (Specification, Interaction and Reconfiguration in Dialogue Understanding Systems), EC Project IST-199910516, and D'Homme (Dialogues in the Home Machine Environment), EC Project IST-2000-26280, and STINT (The Swedish Foundation for International Cooperation in Research and Higher Education). An extended presentation of this work appears in (Larsson, 2002).
}

First, we will give a brief review of Sidner's theory and discuss its merits and drawbacks ${ }^{2}$. We then provide an alternative account based on the concept of Issues Under Negotiation. We explain how IUN can be added to GoDiS, and give an information state analysis of a simple negotiative dialogue. Finally, we draw some conclusions and point to possible future research areas.

\section{Sidner's theory of negotiative dialogue}

As the title says, Sidner's theory is formulated as "an artificial discourse language for collaborative negotiation". This language consists of a set of messages (or message types) with propositional contents ("beliefs"). The effects of an agent transmitting these messages to another agent is formulated in terms of the "state of communication" after the message has been received. The state of communication includes individual beliefs and intentions, mutual beliefs, and two stacks for Open Beliefs and Rejected Beliefs. Some of the central messages are

- ProposeForAccept (PFA agt1 belief agt2): agt1 expresses belief to agt2.

- Reject (RJ agt1 belief agt2): agt1 does not believe belief, which has been offered as a proposal

\footnotetext{
${ }^{2}$ A more in-depth description of Sidner's account and its relation to the GoDiS model, including a reformulation of Sidner's artificial negotiation language in terms of GoDiS information state updates, can be found in (Cooper et al., 2001).
} 
- AcceptProposal (AP agt1 belief agt2): agt 1 and agt 2 now hold belief as a mutual belief

- Counter

(CO agt1 belief1 agt2 belief2): Without rejecting belief1, agt 1 offers belief 2 to agt2

In addition, there are three kinds of acknowledgement messages, the most important being AcknowledgeReceipt (AR agt1 belief agt2), which may occur after a ProposeForAccept message and results in belief being pushed on the stack for Open Beliefs. Acknowledgement indicates that a previous message from agt2 about belief has been heard; the agents will not hold belief as a mutual belief until an AcceptProposal message has been sent.

While we will not give a detailed analysis of the effects of each of these acts, some observations are important for the purposes of this paper. Specifically, a counter-proposal (CO agt1 belief1 agt2 belief2) is analyzed as a composite message consisting of two PFA messages with propositional contents. The first proposed proposition is belief2 (the "new" proposal), and the second is (Supports (Not belief1) belief2), i.e. that belief2 supports the negation of belief1 (the "old" proposal). Exactly what is meant by "supports" here is left unspecified, but perhaps logical entailment is at least a simple kind of support.

- (PFA agt1 belief2 agt2) - (PFA agt1 (Supports (Not belief1)
belief2) agt2)

Sidner's analysis of proposals is only concerned with propositional contents. A Request for action is modelled as a proposal whose content is of the form (Should-Do Agt Action). A question is a proposal for the action to provide certain information. This brings us to our first problem with Sidner's account.

\section{Problem 1: Negotiation vs. utterance acceptance}

In Sidner's theory, all dialogue is negotiative in the sense that all utterances (except acceptances, rejections, and acknowledgements) are seen as proposals. This is correct if we consider negotiation as possibly concerning meta-aspects of the dialogue. Since any utterance (content) can be rejected, all utterances can indeed be seen as proposals.

(Clark, 1996) provides a "ladder" with four levels of comprehension involved in grounding of natural language utterances in dialogue.

1. A attends to B's utterance

2. A perceives B's utterance

3. A understands B's utterance

4. A accepts or rejects B's utterance

So in one sense of "negotiative", all dialogue is negotiative since assertions (and questions, instructions etc.) can be rejected or accepted. But some dialogues are negotiative in another sense, in that they contain explicitly discussions about different solutions to a problem. Negotiation, on this view, is distinct from Clark's level 4.

There is thus a stronger sense of negotiation which is not present in all dialogue. A minimum requirement on negotiation in this stronger sense could be that several alternative solutions (answers) to a problem (question or issue) can be discussed and compared before a solution is finally settled on. Sidner is aware of this aspect of negotiation, and notes that "maintaining more than one open proposal is a common feature of human discourses and negotiations." What we want to do is to find a way of capturing this property independently of grounding and of other aspects of negotiation, and use it as a minimal requirement on any dialogue that is to be regarded as negotiative.

On our view, utterances realizing proposalmoves are moves on the same level as other dialogue moves: greetings, questions, answers etc., and can thus be accepted or rejected on this 
level. Accepting a proposal-move on the grounding level merely means accepting the content of the move as a proposal, i.e. as a potential answer to a question. This is different from accepting the proposed alternative as the actual solution to a problem (answer to a question).

To give a concrete example of these different concepts of negotiativity, we can compare the dialogues in Figures 1 and 2. The type negotiation in 1 concerns acceptance-level grounding of the utterance and its content. By contrast, the type of negotiation in 2 concerns domain-level issues rather than some aspect of grounding. We won't have much to say about grounding-related negotiation in this paper, but see (Lewin et al., 2000) for an account of negotiation related to utterance grounding.

\section{Problem 2: Alternatives and counterproposals}

When analyzing a travel agency dialogue (Sidner, 1994b), the travel agent's successive proposals of flights are seen as counterproposals to his own previous proposals, each modelled as a proposition. The difference between proposals and counterproposals is that the latter not only make a new proposal but also proposes the proposition that the new proposal conflicts with the previous proposal (by supporting the negation of the previous proposal). This can be seen as an attempt by Sidner to establish the connection between the two proposals as somehow concerning the same issue.

This analysis is problematic in that it excludes cases where alternatives are not mutually exclusive, which is natural when e.g. booking a flight (since the user presumably only want one flight) but not e.g. when buying a CD (since the user may want to buy more than one). Also, it seems odd to make counterproposals to your own previous proposals, especially since making a proposal commits you to intending the addressee to accept that proposal rather than your previous ones. In many cases (including travel agencies) it seems that the agent may often be quite indifferent to which flight the user selects. Travel agents may often make several proposals in one utterance, e.g. "There is one flight at 7:45 and one at 12:00", in which case it does not make sense to see "one at 12:00" as a counterproposal as Sidner defines them.

Of course, one would not want to use the term "counterproposal" in these cases; what we need is some way of proposing alternatives without seeing them as counterproposals. The basic problem seems to be that when several proposals are "on the table" at once, one needs some way of representing the fact that they are not independent of each other. Sidner does this by adding propositions of the form (Supports (Not belief1) belief2) to show that belief1 and belief2 are not independent; however, this proposition not only claims that the propositions are somehow dependent, but also that they are (logically or rhetorically) mutually exclusive. In our view, this indicates a need for a theory of negotiation which makes it possible to represent several alternatives as somehow concerning the same issue, independently of rhetorical or logical relations between the alternatives. Negotiation, in our view, should not in general be seen in terms of proposals and counterproposals, but in terms of proposing and choosing between several alternatives.

\section{$5 \quad$ Negotiation as discussing alternatives}

In this section, we will attempt to provide a more detailed description of negotiative dialogue. Clearly, negotiation is a type of problemsolving (Di Eugenio et al., 1998). We define negotiative dialogue more specifically to be $d i$ alogue where DPs ${ }^{3}$ discuss several alternative solutions to a problem (issue) before choosing one of them. In line with our issue-based approach to dialogue management, we propose to model negotiable problems (issues) semantically as questions and alternative solutions as alternative answers to a question.

We also propose to keep track of these issues under negotiation and the answers being considered as potential solutions to each issue in the form of a stack (or ordered set) of pairs of issues

\footnotetext{
${ }^{3} \mathrm{DPs}=$ Dialogue Participants
} 
A $>$ Today is January 6th.

propose proposition

$\mathrm{B}($ alt. 1) $>$ Uhuh

accept proposition

$\mathrm{B}($ alt. 2) $>$ No, it's not!

reject proposition

Figure 1: Negotiating utterance (content) acceptance

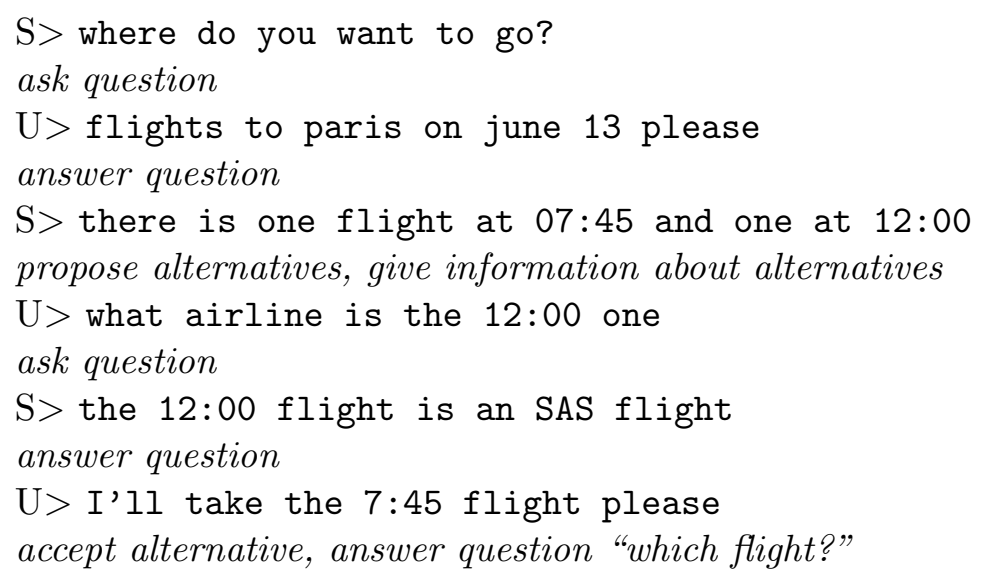

Figure 2: Negotiating alternative solutions to an issue

and sets of answers. We label this stack Issues Under Negotiation, or IUN.

\subsection{Degrees of negotiativity}

Starting from this definition, we can distinguish between fully negotiative dialogue and seminegotiative dialogue. In non-negotiative dialogue, only one alternative can be discussed. In semi-negotiative dialogue, a new alternative can be introduced by revising parameters of the previous alternative; however, previous alternatives are not retained. Finally, in negotiative dialogue: several alternatives can be introduced, and old alternatives are retained and can be returned to.

Semi-negotiative information-oriented dialogue does not require keeping track of several alternatives. All that is required is that information is revisable, and that new database queries can be formed from old ones by replacing some piece of information. This property is implemented in a limited way for example in the Swedish railway information system (a vari- ant of the Philips system (Aust et al., 1994)) which after providing information about a trip will ask the user "Do you want an earlier or later train?". This allows the user to modify the previous query (although in a very limited way) and get information about further alternatives. However, it is not possible to compare the alternatives by asking questions about them; indeed, there is no sign that information about previous alternatives is retained in the system.

\subsection{Factors influencing negotiation}

There are a number of aspects of the dialogue situation which affect the complexity of negotiative dialogues, and allows further subclassification of them. This sub-classification allows us to pick out a subspecies of negotiative dialogue to implement.

On our definition, negotiation does not require conflicting goals or interests, and for this reason it may not correspond perfectly to the everyday use of the word "negotiation". However, we feel it is useful to keep collaborativity 
(i.e. lack of conflicting goals) as a separate dimension from negotiation. Also, it is common practice in other fields dealing with negotiation (e.g. game theory, economy) to include collaborative negotiation (cf. (Lewin et al., 2000)).

A second factor influencing negotiation is the distribution of information between DPs. In some activities, information may be symmetrically distributed, i.e. DPs have roughly the same kind of information, and also the same kind of information needs (questions they want answered). This is the case e.g. in the Coconut (Di Eugenio et al., 1998) dialogues where DPs each have an amount of money and they have to decide jointly on a number of furniture items to purchase. In other activities, such as a travel agency, the information and information needs of the DPs is asymmetrically distributed. The customer has access to information about her destination, approximate time of travel etc., and wants to know e.g. exact flight times and prices. The travel agent has access to a database of flight information, but needs to know when the customer wants to leave, where she wants to travel, etc.

A third variable is whether DPs must commit jointly (as in e.g. the Coconut dialogues) or one DP can make the commitment by herself (as e.g. in flight booking). In the latter case, the acceptance of one of the alternatives can be modelled as an answer to an issue on IUN by the DP responsible for the commitment, without the need for an explicit agreement from the other DP. In the former case, a similar analysis is possible, but here it is more likely that an explicit expression of agreement is needed from both DPs. This variable may perhaps be referred to as "distribution of decision rights".

Travel agency dialogue, and dialogue in other domains with clear differences in information and decision-right distribution between roles, has the advantage of making dialogue move interpretation easier since the presence of a certain bits of information in an utterance together with knowledge about the role of the speaker and the role-related information distribution often can be used to determine dialogue move type. For example, an utterance containing the phrase "to
Paris" spoken by a customer in a travel agency is likely to be intended to provide information about the customer's desired destination.

\section{Issues Under Negotiation (IUN)}

In this section we discuss the notion of Issues Under Negotiation represented by questions, and how proposals relate to issues on IUN. We also discuss how this approach differs from Sidner's.

\subsection{Negotiable issues and activity}

Which issues are negotiable depends on the activity. For example, it is usually not the case that the name of a DP is a negotiable issue; this is why it would perhaps seem counterintuitive to view an introduction ("Hi, my name is NN") as a proposal. However, it cannot be ruled out that there is some activity where even this may become a matter of negotiation. Also, it is usually possible in principle to make any issue into a negotiable issue, e.g. by raising doubts about a previous answer.

However, for our current purposes we may make a distinction between negotiable and nonnegotiable issues in an activity. The advantage of this is that the more complex processing and domain-specific knowledge required for negotiable issues are only required for issues which the system needs to be able to negotiate. The drawback, of course, is that the system becomes less flexible; however, there is always the possibility of defining all issues as negotiative if one so desires.

\subsection{Alternatives as answers to issues on IUN}

Given that we analyze Issues Under Negotiation as questions, it is a natural move to analyze the alternative solutions to this issue as potential answers. On this view, a proposal has the effect of adding an alternative answer to the set of alternative answers to an issue on IUN. An answer to the question on IUN is equivalent to accepting one of the potential answers as the actual answer. That is, a question on IUN is resolved when an alternative answer is accepted. 
Here we see how our concept of acceptance differs from Sidner. On our view a proposal can be accepted in two different ways: as a proposal, or as the answer to an issue on IUN. Accepting a proposal move as adding an alternative corresponds to meta-level acceptance. However, accepting an alternative as the answer to an issue on IUN is different from accepting an utterance. Given the optimistic approach to acceptance, all proposals will be assumed to be accepted as proposals; however, it takes an answer-move to get the proposed alternative accepted as the solution to a problem.

\section{Adding IUN to the GoDiS information state}

The ideas described in this paper are currently being implemented in GoDiS (Bohlin et al., 1999), an experimental dialogue system initially adapted for the travel agency domain but later adapted for several other domains. GoDiS is implemented using the TrindiKIT(Larsson and Traum, 2000; Larsson et al., 2000), a toolkit for experimenting with information states and dialogue move engines and for building dialogue systems.

The notion of information state used by GoDiS is basically a version of the dialogue game board which has been proposed by (Ginzburg, 1998). We represent information states of a dialogue participant as a record of the type shown in Figure 3.

The main division in the information state is between information which is private to the agent and that which is shared between the dialogue participants. The private part of the information state contains a PLAN field holding a dialogue plan, i.e. is a list of dialogue actions that the agent wishes to carry out. The AGENDA field, on the other hand, contains the short term goals or obligations that the agent has, i.e. what the agent is going to do next. We have included a field TMP that mirrors the shared fields. This field keeps track of shared information that has not yet been confirmed as grounded, i.e. as having been understood by the other dialogue participant. The SHARED field is divided into a local and a global part. The local part contains information about the latest utterance, and information which may be relevant for interpreting it. The first subfield is for a stack of questions under discussion (QUD). These are questions that can currently be answered elliptically. The LU field contains information about the speaker of, and the moves performed in, latest utterance.

The global part contains shared information which reflects the global state of the dialogue. It contains a set of propositions (commitments) which the agent assumes have been jointly committed to in the dialogue (COM). In order to include Issues Under Negotiation and alternative answers to issues on IUN in the information state, we have also added a new information state field of type OpenStack(Pair(Question,Set(Answer)) $)^{4}$.

We define update rules for updating the information state based on the recognized move(s). The rules are defined in terms of preconditions and effects on the information state; the effects are a list of operations to be executed if the preconditions are true.

Regarding the semantics of questions, propositions, and short answers, we use a simplified version of first order logic with the addition of lambda abstracts for representing questions. Questions and answers can be combined to form propositions. For example, the content of "when do you want to leave?" can be represented as ?x.desired_dept_time(x), the answer "twelve thirty" as $\mathbf{1 2 : 3 0}$, and the proposition resulting from combining the two desired_dept_time(12:30). As a further example, the proposition that a certain flight (denoted f1) departs at 7:45 is represented as dept_time(f1,0745). For a more comprehensive description of the semantics used, see (Larsson, 2002).

\footnotetext{
${ }^{4}$ The choice of a stack is motivated by the fact that several issues may, in principle, be under negotiation at once, and that some issues may be subordinate to others. An open stack is a stack where non-topmost elements are accessible for inspection and deletion.
} 


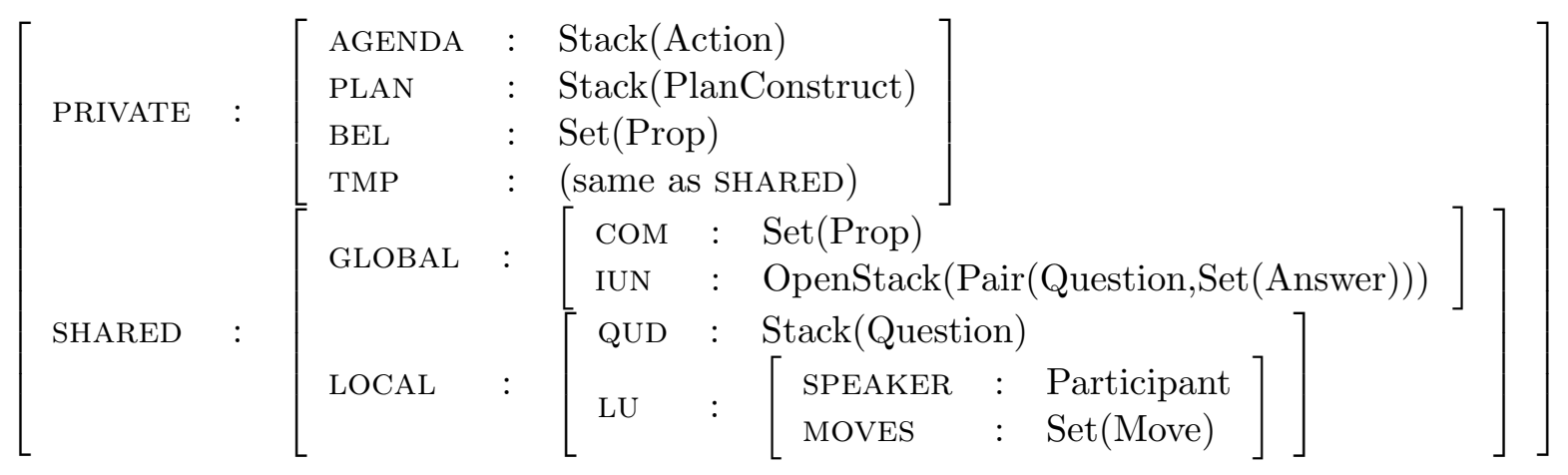

Figure 3: Extended GoDiS information state type, including IUN

\section{An example}

In the (invented) example in 4, the question on IUN is ? $x$.desired_flight $(x)$, i.e. "Which flight does the user want?". The user supplies information about her desired destination and departure date; this utterance is interpreted as a set of answer-moves by the system since it provides answers to questions that the system has asked or was going to ask. As a response to this, GoDiS performs a database search which returns two flights $\mathbf{f} 1$ and $\mathbf{f} 2$ matching the specification, and stores the database results in PRIVATE.BEL. The system then proposes these flights as answers to the IUN. The system also supplies some information about them. As a result, the IUN is now associated with two alternative answers, f1 and f2. Finally, the user provides an answer to the question on IUN, thereby accepting one of these alternatives as the flight she wants to take.

Because of space limitations, this dialogue does not include any discussion or comparison of alternatives, but it could easily be extended to cover e.g. the dialogue in 2.

In a travel agency setting, it can be argued that the informational distribution and decision rights associated with the roles of customer and clerk in a travel agency are sufficient to distinguish proposals for acceptances, but in a more complex setting the move recognition will require more information about surface form and dialogue context.

\section{Relation to previous work}

Much work on negotiative dialogue, including that of Sidner, is based on variations on the BDI model of classic AI and uses generalised planning and plan recognition as an integral part of dialogue processing (e.g. (Cohen and Levesque, 1991), (Grosz and Kraus, 1993) (Chu-Carroll and Carberry, 1994)). The risk with this kind of very general reasoning is that it may become computationally complex and expensive. Therefore, we believe it is useful to start with a simple theory involving reasoning specific to a certain kind of dialogue and see how far that takes us, and at what point general planning and reasoning is really necessary.

In working on GoDiS our strategy has been to start from a basic issue-based approach and gradually extending it, while trying to keep things as simple as possible. We believe that the issue-based approach can be extended with, and is compatible with, planning and general reasoning mechanisms. This is also in line with the idea behind the information state update approach as implemented in TRINDIKIT, i.e. to allow for experimentation with different kinds of information states and information state update strategies at various levels of complexity (rather than being confined to choosing between oversimplified finite state / frame-based approaches on the one hand, or complex plan-based approaches on the other).

Also, most of the plan-based dialogue research is focused on relatively complex dialogues involving collaborative planning. The model presented here is not committed to the view that negotiation only takes place in the context of collaborative planning. In the sense of negotiative dialogue used here, i.e. dialogue involving sev- 
A $>$ flights to paris, june 13

answer(desired_dest_city(paris))

answer(desired_dept_date(13/6))

B $>$ OK, there's one flight leaving at 07:45 and one at 12:00

propose $(\mathbf{f} \mathbf{1})$

propose $(\mathbf{f} 2)$

inform(dept_time(f1,07:45))

inform(dept_time(f2,12:00))

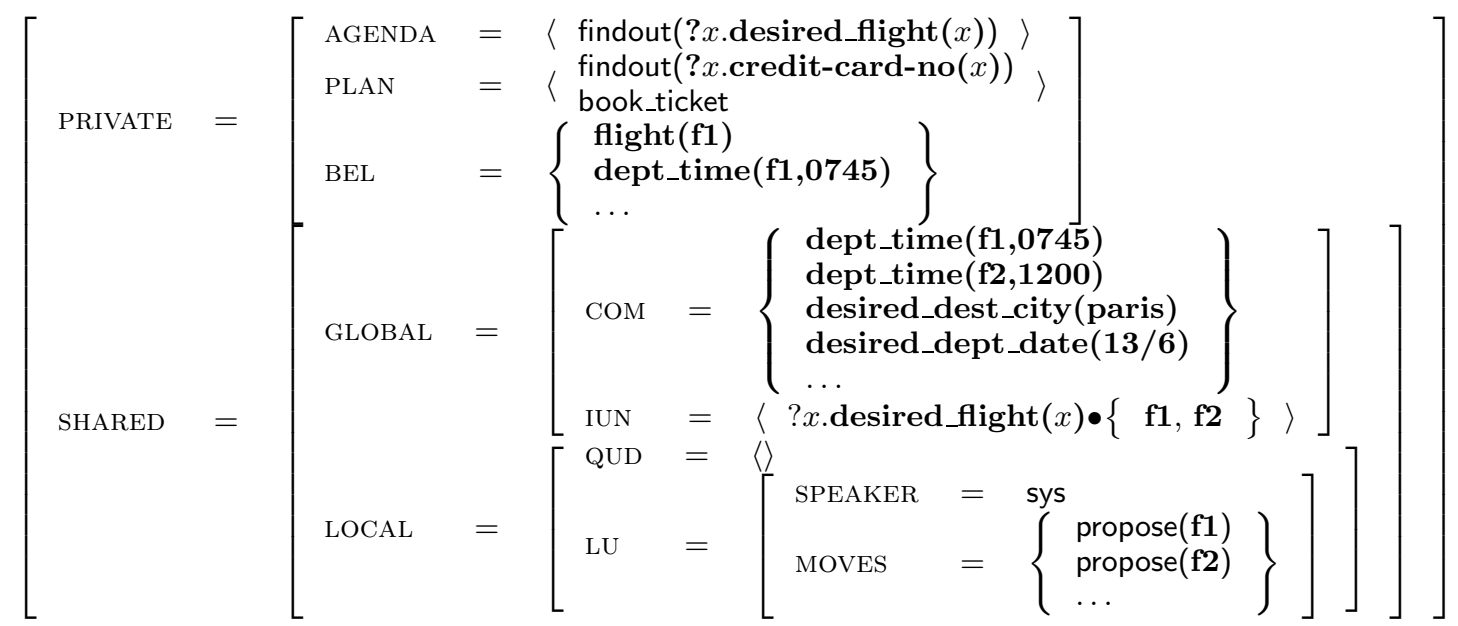

A> I'll take the 07:45 one

answer(desired_flight $(X) \&$ dept_time $(X, 07: 45))$

(after contextual interpretation: answer(desired_flight(f1)))

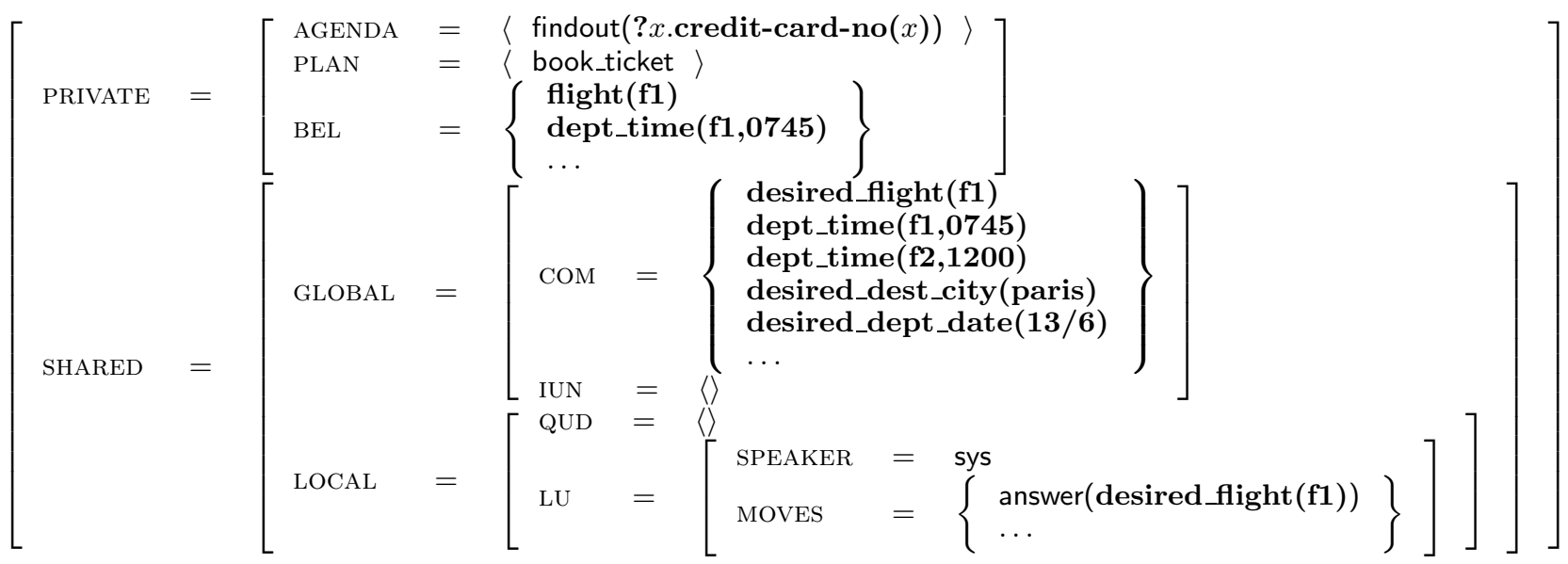

Figure 4: Example dialogue 
eral alternative solutions to some problem, negotiation may also concern matters of fact. This can be useful e.g. in tutorial dialogue where a tutor asks a question, gives some alternative answers, and the student's task is to reason about the different alternatives and decide on one of them. In the travel agency domain, it is often not necessary to explicitly represent e.g. the fact that the deciding on a flight is a precondition of a general plan for traveling; instead, we can represent it simply as a fact concerning which flight the user wants to take.

(Rosé et al., 1995) provide an account of discourse structure for dialogues involving several alternatives (or "threads"), which appears to be compatible with the view presented here. However, the focus on discourse structures rather than information states and dialogue processing (in terms of information state updates) makes this work less relevant to dialogue systems design, at least from the perspective of an information state update approach.

\section{Conclusions and future work}

On our approach, an Issue Under Negotiation is represented as a question, e.g. what flight the user wants. In general, this means viewing problems as issues and solutions as answers. This approach has several advantages. Firstly, it provides a straightforward an intuitively sound way of capturing the idea that negotiative dialogue involves several alternative solutions to some issue or problem, and that proposals introduce such alternatives. Secondly, it distinguishes two types of negotiation (dialogue negotiation and issue negotiation) and clarifies the relation between them. Thirdly, since this account is a natural extension of the general ideas behind the GoDiS system (e.g. using issues as a basis for dialogue management), it allows the use of independently motivated theory and machinery to handle a new problem. Apart from implementing the theory described in this paper, possible future work includes applying the theory to new domains and extending it to handle more complex kinds of negotiation, possibly making use of work on collaboration and social action such as (Castelfranchi et al., 2000).

\section{References}

H. Aust, M. Oerder, F. Seide, and V. Steinbiss. 1994. Experience with the Philips automatic train table information system. In Proc. of the 2nd Workshop on Interactive Voice Technology for Telecommunications Applications (IVTTA), pages 67-72, Kyoto, Japan.

P. Bohlin, R. Cooper, E. Engdahl, and S. Larsson. 1999. Information states and dialogue move engines. In J. Alexandersson, editor, IJCAI-99 Workshop on Knowledge and Reasoning in Practical Dialogue Systems, pages 25-32.

C. Castelfranchi, F. Dignum, C. Jonker, and J. Treur. 2000. Deliberate normative agents: Principles and architecture. In Jennings and Lesperance (Jennings and Lesperance, 2000), pages 206-220.

Jennifer Chu-Carroll and Sandra Carberry. 1994. A plan-based model for response generation in collaborative task-oriented dialogues. In $A A A I$, Vol. 1, pages 799-805.

H. H. Clark. 1996. Using Language. Cambridge University Press, Cambridge.

Phillip R. Cohen and Hector J. Levesque. 1991. Teamwork. Nous, 35.

Robin Cooper, Stina Ericsson, Staffan Larsson, and Ian Lewin. 2001. An information state update approach to collaborative negotiation. In Peter Kühnlein, Hannes Rieser, and Henk Zeevat, editors, BI-DIALOG 2001-Proceedings of the 5th Workshop on Formal Semantics and Pragmatics of Dialogue, pages 270-9, http: //www . uni-bielefeld.de/BIDIALOG, June, 14th-16th. ZiF, Univ. Bielefeld.

B. Di Eugenio, P.W. Jordan, R.H. Thomason, and J.D. Moore. 1998. An empirical investigation of proposals in collaborative dialogues. In Proceedings of ACL-COLING 98: 36th Annual Meeting of the Association of Computational Linguistics and 17th International Conference on Computational Linguistics, pages 325-329.

J. Ginzburg. 1998. Clarifying utterances. In J. Hulstijn and A. Niholt, editors, Proc. of the Twente Workshop on the Formal Semantics and Pragmatics of Dialogues, pages 11-30, Enschede. Universiteit Twente, Faculteit Informatica.

Barbara [J.] Grosz and Sarit Kraus. 1993. Collaborative plans for group activities. In Proceedings IJCAI-93, pages 367-373. 
N. Jennings and Y Lesperance, editors. 2000. Proceedings of the 6th International Workshop on Agent Theories, Architectures, and Languages (ATAL'1999), Springer Lecture Notes in AI 1757. Springer Verlag, Berlin.

Staffan Larsson and David Traum. 2000. Information state and dialogue management in the trindi dialogue move engine toolkit. NLE Special Issue on Best Practice in Spoken Language Dialogue Systems Engineering, pages 323-340.

Staffan Larsson, Alexander Berman, Johan Bos, Leif Grönqvist, Peter Ljunglöf, and David Traum. 2000. Trindikit 2.0 manual. Technical Report Deliverable D5.3 - Manual, Trindi.

Staffan Larsson. 2002. Issue-based Dialogue Management. Ph.D. thesis, Göteborg University.

Ian Lewin, Robin Cooper, Stina Ericsson, and C.J. Rupp. 2000. Dialogue moves in negotiative dialogues. Project deliverable 1.2, SIRIDUS.

C. P. Rosé, B. Di Eugenio, L. S. Levin, and C. Van Ess-Dykema. 1995. Discourse processing of dialogues with multiple threads. In Proc. $A C L$, Boston, MIT, June.

Candace L. Sidner. 1994a. An artificial discourse language for collaborative negotiation. In Proceedings of the forteenth National Conference of the American Association for Artificial Intelligence (AAAI-94), pages 814-819.

Candace. L. Sidner. 1994b. Negotiation in collaborative activity: A discourse analysis. KnowledgeBased Systems, 7(4):265-267. 